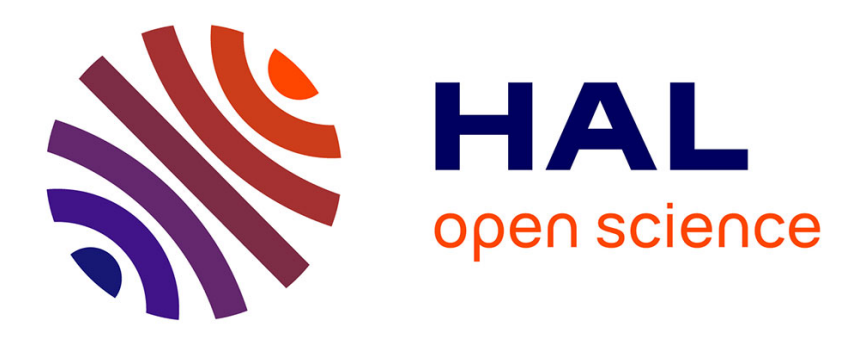

\title{
Fostering Design Process of Shape-Changing Interfaces Hyunyoung Kim
}

\section{To cite this version:}

Hyunyoung Kim. Fostering Design Process of Shape-Changing Interfaces. 2021. hal-03223766

\author{
HAL Id: hal-03223766 \\ https://hal.science/hal-03223766 \\ Preprint submitted on 11 May 2021
}

HAL is a multi-disciplinary open access archive for the deposit and dissemination of scientific research documents, whether they are published or not. The documents may come from teaching and research institutions in France or abroad, or from public or private research centers.
L'archive ouverte pluridisciplinaire HAL, est destinée au dépôt et à la diffusion de documents scientifiques de niveau recherche, publiés ou non, émanant des établissements d'enseignement et de recherche français ou étrangers, des laboratoires publics ou privés. 


\title{
Fostering Design Process of Shape-Changing Interfaces
}

\author{
Hyunyoung Kim \\ Université Grenoble Alpes \\ Grenoble, France \\ hyunyoung.kim@univ-grenoble-alpes.fr
}

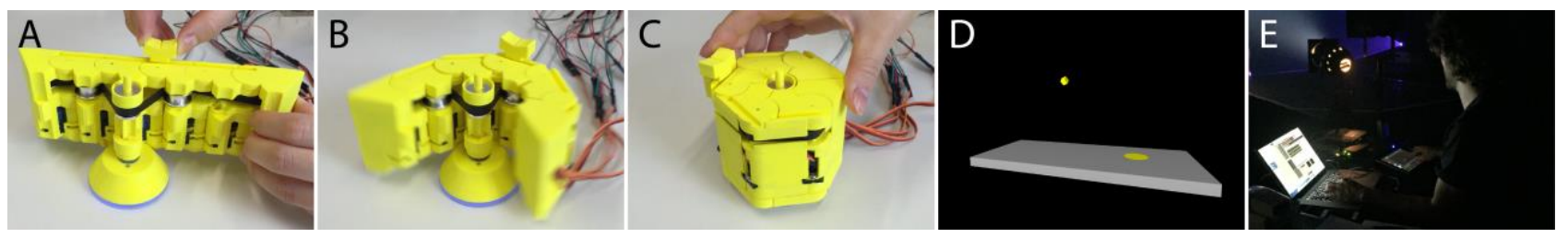

Figure 1. (A, B, C) KnobSlider is a shape-changing device that changes shape between a slider and a knob to accommodate user needs. For example, when a light engineer changes light intensity of a projector on a stage (D), he can use it as a slider (A). He can then press the central button to change the shape $(B)$ into a knob $(C)$ to move the position of the projected light. The design of KnobSlider was based on contextual interviews with eight professional users including a light artist (E).

\begin{abstract}
Shape-changing interfaces match forms and haptics with functions and bring affordances to devices. I believe that shape-changing interfaces will be increasingly available to end-users in the future. To increase acceptance of shapechanging interfaces by end-users, we need to provide designers with design criteria and framework closely grounded on their current skills and needs. Also, we need to provide them with prototyping tools to enable quick assessment of ideas in the physical world. In this paper, I introduce the three threads of my Ph.D. research in the direction of providing the design tools. First, I advance existing shape-changing interface taxonomies to broaden design vocabulary and systemize design framework, based on the classification of everyday objects. Second, I conduct a study with end-users to suggest interaction techniques and design guidelines for shape-changing interfaces from their current practice. Lastly, I develop a physical prototyping tool for shape-changing interfaces to shorten prototyping iterations based on well-known Lego-like bricks.
\end{abstract}

\section{Author Keywords}

Shape-changing interfaces; tangible user interfaces.

\section{INTRODUCTION}

Shape-changing interfaces will radically change end-users' interactions with computers, by providing physical affordances beyond flat touchscreens and by allowing the

Permission to make digital or hard copies of part or all of this work for personal or classroom use is granted without fee provided that copies are not made or distributed for profit or commercial advantage and that copies bear this notice and the full citation on the first page. Copyrights for thirdparty components of this work must be honored. For all other uses, contact the Owner/Author.

UIST '18 Adjunct, October 14-17, 2018, Berlin, Germany

(C) 2018 Copyright is held by the owner/author(s).

ACM ISBN 978-1-4503-5949-8/18/10.

https://doi.org/10.1145/3266037.3266131 users to use full dexterity of their bodies. However, current designs of shape-changing interfaces are often driven by technologies rather than user needs. To ensure users' easy acceptance of shape-changing interfaces, we need to enable designers to systemically explore shape-changing interface ideas and evaluate them.

As a tool to systemically explore ideas, previous works suggest designing by using a shape-changing interface taxonomy [7]. They also extensively evaluate shapechanging interfaces with end-users [2] and provide design guidelines for future studies. Or, they provide prototyping tools to allow designers to quickly build and evaluate ideas [3]. In my thesis, I extend this direction of providing tools that can foster a design process.

In my thesis, I explore 3 tools of different nature to help the design of shape changing device: 1) design framework: I use everyday objects to refine existing taxonomies and propose a new framework to describe the possible shapes a device can have. 2) design criteria: I use end-user observations to devise a list of requirements for reconfigurable devices and show how to use them by building shape-changing parameter controls. 3) prototyping tool: I develop a Lego-like physical prototyping toolkit and show how it can physicalize a wide range of shapechanging interface ideas. Throughout the thesis, I try to use interactions and objects that are familiar to end-users or designers, believing that it would increase designers' adoption of such tools.

In this paper, I first expose the grand challenges in shapechanging interface research and how my thesis contributes to them. Then I introduce each piece of my research papers in the spotlight of the problems, contributions, and research methods. 


\section{GRAND CHALLENGES IN}

\section{SHAPE-CHANGING INTERFACE RESEARCH}

Alexander et al. [1] discuss overarching challenges in shape-changing interface research, through a workshop with professional researchers related to shape-changing interfaces. They categorize the challenges into the design, user behavior, technological, and societal challenges.

- Design challenges: designers need to extensively consider different physical forms and dynamics to deliver aesthetic, usable, and safe shape-changing interfaces.

- User behavior challenges: user experience (UX) of shape-changing interfaces should be vigorously investigated to understand in which domains and tasks they are beneficial.

- Technological challenges: implementing shape-changing interfaces requires knowledge of complex electronics and mechanical engineering. The challenges include having prototyping tools to accelerate design iterations, miniaturizing the devices, and integrating with other $\mathrm{I} / \mathrm{O}$ modalities.

- Societal challenges: future regulations should consider ethical risks regarding shape-changing interfaces, as their physicality can potentially harm users or take more natural resources than regular touch displays. This challenge would affect the long-term adoption of shapechanging interfaces.

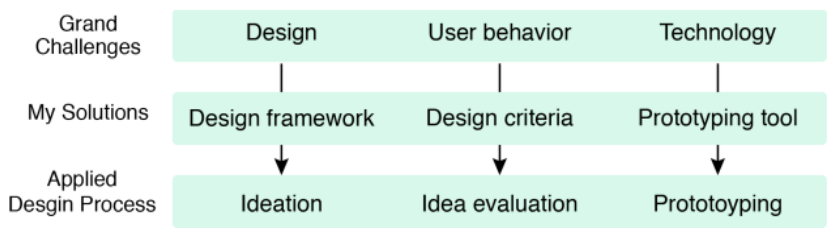

Figure 2. Grand challenges that I contribute and their applications in a design process.

I focus on the first three challenges during my dissertation (Figure 2). Especially I focus on them in a way to lift up a whole design process of shape-changing interfaces. First, the established design framework will enable designers systemically and extensively explore ideas in an ideation phase. Second, studies on user behavior on shape-changing interfaces can provide idea selection criteria in an idea evaluation phase. Lastly, a prototyping tool will reduce time to physically implement the ideas in a prototyping phase.

\section{DESIGN CHALLENGES: DESIGN FRAMEWORK}

In a design process, designers often generate as many ideas as possible, choose the best ideas, and then develop the ideas. Enabling them to systematically and extensively explore design ideas is hence important.

In shape-changing interface research, taxonomies help designers to analyze existing interfaces and generate new ones in a systemic way. Taxonomies by Rasmussen $e t$ al. and Roudaut et al. $[9,10]$ largely contribute to it by focusing on general views on shapes rather than technologies or purposes. Designers used the taxonomies in ideation workshops [7].

\section{Morphees+: Refining Taxonomies for Shape-Changing Interfaces [CHI'18]}

Shape-changing interface taxonomies $[9,10]$ help designers describe the reconfigurability of interfaces and can be used to design new interfaces. To date, there has been little effort to unify and strengthen these taxonomies despite that shapechanging interfaces are continuously evolving. Also, the taxonomies' descriptive power has been hardly tested, and it is uncertain if they are comprehensive and complete to describe all shape-changes.

I propose Morphees+ [5] (Figure 3), a refined taxonomy based on the two most-widely used taxonomies in the community $[9,10]$. The taxonomy refines existing Area and Volume features from the previous taxonomies to Size feature, which has subdivided dimensions - Length, Area, and Volume. The refined taxonomy also includes Modularity, which can now describe the number of possible combinations of modular interfaces, e.g., brick-based interfaces.

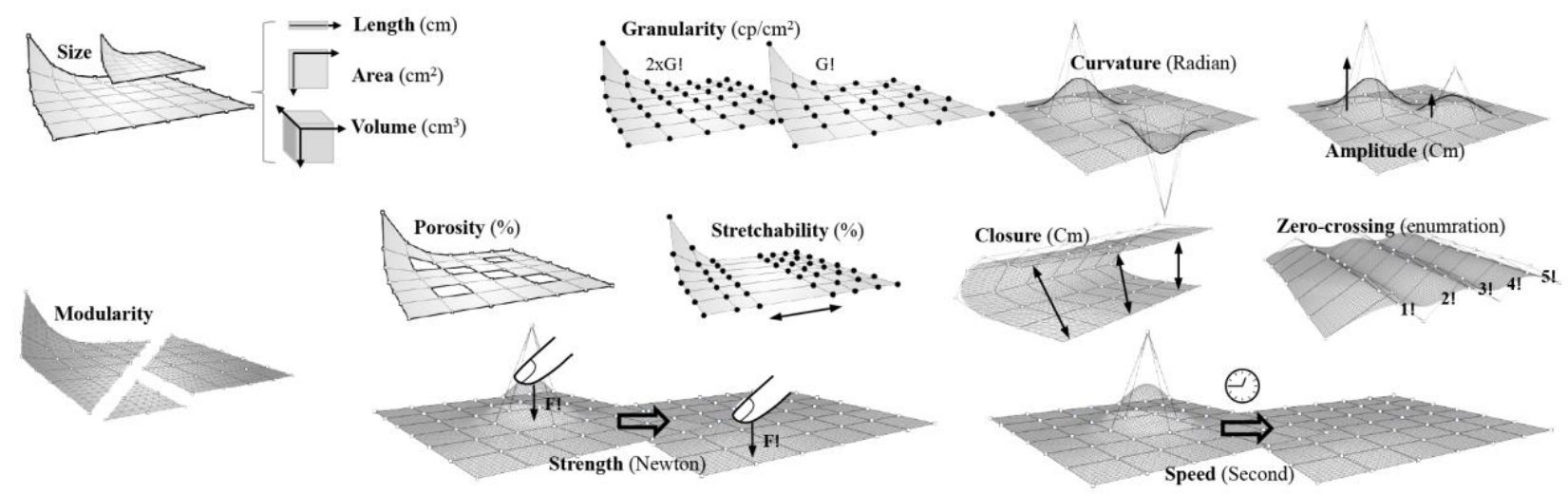

Figure 3. Refinement of the Shape Resolution features after analyzing daily reconfigurable objects. We add (A) Size and Modularity to complete the $(B)$ previous features. Size is an extended feature from the original definition of Area. Modularity is a new feature measuring the ability to be split into several parts that can be recombined [5]. 
To refine the taxonomies, me and my coauthors collected 82 reconfigurable objects such as radio antenna, balloon, and Lego bricks. We then classified them using the two taxonomies and analyzed the data. It revealed that the two taxonomies are largely overlapped, there are rooms for subjective understandings of the features, and there are some features that do not precisely capture shape-changes. Based on these findings, we refined the taxonomies by adding or refining features. We also analyzed the materials of the reconfigurable objects to inform how to implement reconfigurable interfaces.

Reconfigurable daily objects have been used to inspire new shape-changing interfaces and to quickly evaluate interaction ideas before implementing working prototypes. This paper is the first systematic study of reconfigurable everyday objects for shape-changing interfaces. The collection and analysis of the 82 objects informed the design space of shape-changing interfaces. The refined taxonomy will better support designers to generate ideas and communicate about their designs.

\section{USER BEHAVIOR CHALLENGES: DESIGN CRITERIA}

I refer to shape-changing controls as input interfaces that are manually or automatically reconfigurable. They are designed in a tempt to replace conventional physical input interfaces such as knobs and sliders. I explore two use cases of reconfigurable controls: 1) having both flexibility and tangibility, and 2) increasing users' input throughput [11]. The two studies aim to provide design guidelines for shapechanging controls in certain contexts. I follow design processes and discuss the devices benefits and how they should be improved for future studies.

\section{KnobSlider: Design Criteria for Having Both Flexibility} and Tangibility [CHI'18]

Conventional mixing consoles, cockpit interfaces, camera controller provide great tangibility, allowing eyes-free control, quick grasp of the interfaces, and so on. However, the interfaces are often crowded and bulky to control many parameters on them. Touchscreen interfaces solve the crowdedness problem with time-multiplexing interfaces. However, they lose the benefit of physical interfaces.

I propose KnobSlider [4], a shape-changing interface that can shift to a knob or a slider (see Figure 1) on users' demand. It solves the problem of having only tangibility or flexibility with conventional TUIs and touch screens, by having time-multiplexing (flexibility) on a physical device (tangibility). For instance, when a light engineer wants to change the size of a projected light, he uses the slider state of the device (Figure 1.A). When he wants to move the position of the light across a stage, he switches the state to a knob (Figure 1.B-C) and continues controlling the parameter while keeping his eyes on the stage.

Me and my coauthors designed the device following a design process. First, we conducted a formative study with eight professional users who use parameter controls, mainly knobs and sliders for their work. They included a movie operator, light engineers, pilots, and so on. We observed how they use their interfaces in ecological settings and interviewed them after observations. Through the study, we derived six design requirements for parameter control interfaces. We evaluated primitive new shape-changing device ideas based on the requirements and implemented the final design KnobSlider. We then brought the device back to the users gathered their feedback to evaluate it.

Through this study, we derived the six requirements as design criteria for flexible parameters control interfaces, which can be used in future studies. The working prototype KnobSlider combines the benefits of a physical knob and a slider through on-demand shape change. The evaluation of the KnobSlider with end-users revealed that we need to consider perceptional aspects and safety of shape-changing interfaces, which is reassuring the user behavior challenge.

\section{SplitSlider: Design Criteria for Uncertainty Input TUls [To be submitted]}

As processing large data gets attention, getting more accurate input from users is getting important. Users can face uncertainty when they produce data, e.g., when they enter their weight to dose a medicine, a time frame to search for a flight, and their satisfaction at an airport service.

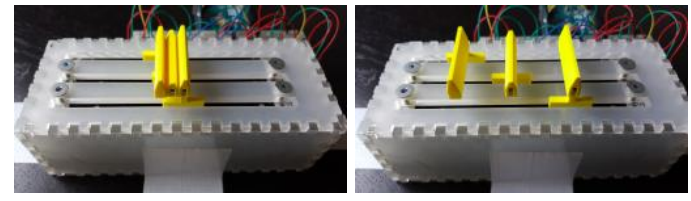

Figure 4. Working prototype of SplitSlider. (a) One thumb as standard input. (b) Separated thumbs for communicating input uncertainty.

I propose SplitSlider, a slider that can have three thumbs for uncertain input and one thumb for certain input (Figure 4). It allows users to switch between certain and uncertain input, using dexterity of their hands. In the study, me and my coauthors explored five low-fidelity devices that allow uncertain input on conventional tangible input interfaces, knobs and sliders. For instance, users can increase a knob diameter to express their uncertainties while rotating it to express values. We conducted a focus group interview to evaluate the devices and derive design criteria for uncertainty input tangible interfaces. We then implemented the working prototype SplitSlider and conducted a user study.

Designers can refer the design requirements when they design shape-changing controls that can input uncertainty and also that can input two parameters simultaneously. The five low-fidelity devices can be used for other multidimensional inputs such as changing the knob diameter to change channels on a mixing board and rotating it to change the values of the channels. It will increase users' input throughput on physical interfaces. 


\section{TECHNOLOGICAL CHALLENGES: PROTOTYPING TOOL}

Currently, implementing working prototypes is the biggest bottleneck in the design process of shape-changing interfaces. To solve the problem, researchers have provided designers with physical prototyping tools that can quickly implement structures and motions and evaluate ideas. Modular physical tools especially allow complex structures through repetitive yet simple assembly processes. For instance, designers can assemble physical struts [6], clips [3], or primitives [8] to prototype shape-changing interfaces. Future modular toolkits should be able to implement full lexical of reconfigurable structures such as the taxonomies $[9,10]$. It would require lots of different types of elements rather than one or a few. Ideally, it should be easy to assemble, and the implementation of the toolkit should be easy and accessible to DIY communities, which will encourage designers to implement and use the toolkits.

\section{BriCAD: a Brick-Based Physical Prototyping Tool [To be submitted]}

To allow designers to fully explore design vocabularies for shape-changing interfaces, I previously suggested refined taxonomy Morphees+ [5]. However, the taxonomy would not be enough to evaluate shape-changing interface ideas and hopefully allow any DIY makers make their own shape-changing interfaces. While 3D printers and other fabrication tools are wide spreading, building shapechanging interfaces still require substantial modeling skills and design iterations. 3D printing and combining actuation electronics can take many hours and significantly slow down design processes.

I propose BriCAD, consisting of a set of bricks and a base in charge of the actuating and sensing shape-changes. The bricks have different lexica such as changing Size or Curvature, which are features from the Morphees+ [5]. It will allow designers to quickly implement shape-changing interface ideas and evaluate them without 3D modeling or printing. To utilize the BriCAD, designers can assemble the bricks together and put them on the base. The motors in the base can actuate shape-change of the bricks, hence the designers can evaluate their ideas with temporal figures such speed of overall shape-changes or trajectories of movements. When designers like the idea and want to change the design for a better look, the base can scan the structure of the bricks and send it to CAD software. It will give swift transitions for designers to continue editing the sent 3D models, printing, and connecting them to electronics for further evaluations.

\section{DISSERTATION STATUS}

I plan to submit my dissertation in fall 2019, hence the UIST Doctoral Symposium will provide me a great opportunity to sharpen my contributions to shape-changing interface research. I expect feedbacks on my methodology throughout the thesis and how to tie the individual projects closer. The current project of the physical prototyping tool would also benefit from feedbacks on the evaluation method and further applications.

\section{REFERENCES}

1. Jason Alexander, Anne Roudaut, Jürgen Steimle, Kasper Hornbæk, Miguel Bruns Alonso, Sean Follmer, and Timothy Merritt. Grand Challenges in ShapeChanging Interface Research. CHI '18.

2. Erik Grönvall, Sofie Kinch, Marianne Graves Petersen, and Majken K. Rasmussen. Causing commotion with a shape-changing bench: experiencing shape-changing interfaces in use. CHI '14.

3. John Hardy, Christian Weichel, Faisal Taher, John Vidler, and Jason Alexander. ShapeClip: Towards Rapid Prototyping with Shape-Changing Displays for Designers. CHI '15.

4. Hyunyoung Kim, Céline Coutrix, and Anne Roudaut. KnobSlider: Design of a Shape-Changing UI for Parameter Control. CHI '18.

5. Hyunyoung Kim, Celine Coutrix, and Anne Roudaut. Morphees+: Studying Everyday Reconfigurable Objects for the Design and Taxonomy of Reconfigurable UIs. CHI ' 18.

6. Danny Leen, Raf Ramakers, and Kris Luyten. StrutModeling: A Low-Fidelity Construction Kit to Iteratively Model, Test, and Adapt 3D Objects. UIST '17.

7. Mie Nørgaard, Tim Merritt, Majken Kirkegaard Rasmussen, and Marianne Graves Petersen. Exploring the design space of shape-changing objects: imagined physics. DPPI '13.

8. Hayes Solos Raffle, Amanda J. Parkes, and Hiroshi Ishii. Topobo: a constructive assembly system with kinetic memory. CHI '04.

9. Majken K. Rasmussen, Esben W. Pedersen, Marianne G. Petersen, and Kasper Hornbæk. Shape-Changing Interfaces: A Review of the Design Space and Open Research Questions. CHI '12.

10. Anne Roudaut, Abhijit Karnik, Markus Löchtefeld, and Sriram Subramanian. Morphees: toward high "shape resolution" in self-actuated flexible mobile devices. CHI '13.

11. C. E. Shannon. 2001. A mathematical theory of communication. SIGMOBILE Mob. Comput. Commun. Rev. 5, 1 (January 2001), 3-55. 